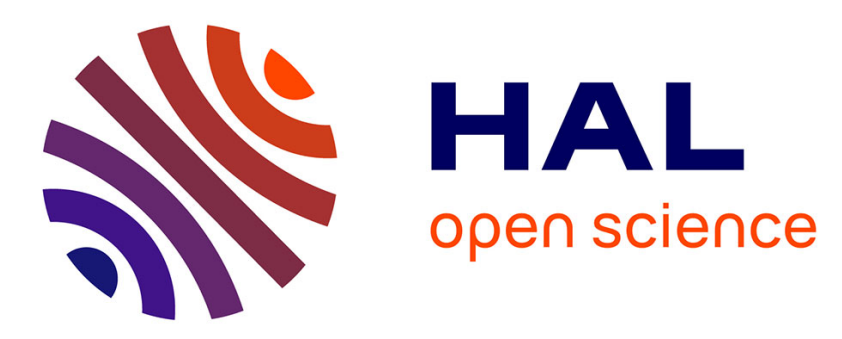

\title{
Stability and Hopf bifurcation for a cell population model with state-dependent delay
}

Mostafa Adimy, Fabien Crauste, Hassan Hbid, Redouane Qesmi

\section{To cite this version:}

Mostafa Adimy, Fabien Crauste, Hassan Hbid, Redouane Qesmi. Stability and Hopf bifurcation for a cell population model with state-dependent delay. SIAM Journal on Applied Mathematics, 2010, 70 (5), pp.1611-1633. 10.1137/080742713 . hal-00542655

\section{HAL Id: hal-00542655 https://hal.science/hal-00542655}

Submitted on 1 Aug 2019

HAL is a multi-disciplinary open access archive for the deposit and dissemination of scientific research documents, whether they are published or not. The documents may come from teaching and research institutions in France or abroad, or from public or private research centers.
L'archive ouverte pluridisciplinaire HAL, est destinée au dépôt et à la diffusion de documents scientifiques de niveau recherche, publiés ou non, émanant des établissements d'enseignement et de recherche français ou étrangers, des laboratoires publics ou privés. 


\title{
STABILITY AND HOPF BIFURCATION FOR A CELL POPULATION MODEL WITH STATE-DEPENDENT DELAY*
}

\author{
MOSTAFA ADIMY ${ }^{\dagger}$, FABIEN CRAUSTE ${ }^{\ddagger}$, MY LHASSAN HBID ${ }^{\S}$, AND \\ REDOUANE QESMI
}

\begin{abstract}
We propose a mathematical model describing the dynamics of a hematopoietic stem cell population. The method of characteristics reduces the age-structured model to a system of differential equations with a state-dependent delay. A detailed stability analysis is performed. A sufficient condition for the global asymptotic stability of the trivial steady state is obtained using a Lyapunov-Razumikhin function. A unique positive steady state is shown to appear through a transcritical bifurcation of the trivial steady state. The analysis of the positive steady state behavior, through the study of a first order exponential polynomial characteristic equation, concludes the existence of a Hopf bifurcation and gives criteria for stability switches. A numerical analysis confirms the results and stresses the role of each parameter involved in the system on the stability of the positive steady state.
\end{abstract}

Key words. hematopoietic stem cells, functional differential equation, state-dependent delay, Lyapunov-Razumikhin function, Hopf bifurcation

AMS subject classifications. 34D20, 34K20, 34K99, 92C37

DOI. $10.1137 / 080742713$

1. Introduction. The production and regulation of blood cells is a very complex process, called hematopoiesis. It involves a population of hematopoietic stem cells (HSCs), able to differentiate and self-renew, in order to maintain an HSC population. These cells produce by differentiation all blood cell lineages (white cells, red blood cells, platelets) that mature within several generations and finally give birth to blood cells that enter the circulating blood.

HSCs are either proliferating or nonproliferating cells. A majority of HSCs is actually in a quiescent stage [18]. Nonproliferating (or resting) HSCs represent a pool of stem cells that is used to produce new blood cells. Proliferating HSCs are actively involved in cell division (growth, DNA synthesis, etc.). The dynamics of HSCs have attracted the attention of modelers for forty years now, due to their involvement in major blood diseases, such as leukemias, that originate at the stem cell level $[27,33]$.

To our knowledge, the first mathematical model of HSC dynamics has been proposed by Mackey [43] in 1978, inspired by Lajtha [40] and Burns and Tannock [17]. Mackey's model consists in a system of two nonlinear delay differential equations, describing the dynamics of proliferating and nonproliferating HSCs. The delay, which is constant, accounts for an average cell cycle duration. Since then, many authors have tried to improve Mackey's model, particularly to better take into account cell

* Received by the editors December 4, 2008; accepted for publication (in revised form) October 15, 2009; published electronically January 13, 2010.

http://www.siam.org/journals/siap/70-5/74271.html

†INRIA Rhône-Alpes, Institut Camille Jordan UMR 5208, 43 blvd du 11 novembre 1918, F-69622 Villeurbanne Cedex, France (mostafa.adimy@inria.fr).

${ }^{\ddagger}$ Université de Lyon, CNRS, Université Lyon 1, Institut Camille Jordan UMR 5208, Batiment du Doyen Jean Braconnier, 43 blvd du 11 novembre 1918, F-69222 Villeurbanne Cedex, France (crauste@math.univ-lyon1.fr).

$\S$ Department of Mathematics, Semlalia Faculty of Sciences, Cadi Ayyad University, P.O. 2390 Marrakesh, Morocco (hbid@ucam.ac.ma).

IDepartment of Mathematics and Statistics, York University, 4700 Keele Street, Toronto, ON, M3J 1P3, Canada (rqesm@mathstat.yorku.ca). 
maturity and cell cycle duration's control in HSC dynamics [1, 8, 46, 47]. This latter point is of particular interest. Cell cycle durations may vary a lot, and the nature of the signal that triggers cell division remains mainly unknown.

In 1978, MacDonald [42] focused on this problem and proposed that cell cycle division times are distributed according to a density function, for instance, a Gamma distribution. In 1993, Mackey and Rey [45] considered a distribution of cell cycle durations according to a density function, but their analysis took into account only a particular case (when the density is a Dirac measure), and then their system exhibited a discrete time delay. For many years, only discrete time delay systems of HSC dynamics were studied; see, for instance, [7, 25, 46, 47] and the references therein. Recently, Adimy and Crauste [1], Adimy, Crauste, and Ruan [5, 6], and Bernard, Bélair, and Mackey [16] analyzed modified versions of Mackey's model [43] by assuming a distribution of cell division times. They stressed the influence of the distribution (some properties of the density function) on solution's behavior. However, this approach is not always satisfactory, especially because no biological data can allow us to precisely determine the nature - the shape - of the age distribution. In 2005, Adimy and Crauste [2] and Adimy, Crauste, and Pujo-Menjouet [4] proposed a model of HSC dynamics in which the cell cycle duration depends on the cell maturity. This is a way of indicating that cell cycles can be shortened for some types of cells, or in particular situations such as diseases or anemias.

In this paper, we focus on the influence of the number of cells on cell cycle durations. Low cell counts lead to quick reactions of the organism, in order to produce enough cells to return to a normal state, and this can then induce shorter cell cycles (this is observed for red cells, where, following an anemia, immature cells enter the bloodstream and replace mature cells very quickly [22]). Moreover, cell cycle duration variability has been the subject of numerous modeling works (see, for instance, Alarcon and Tindall [12], Fuss et al. [28], and Tyson and Novak [58]) based on the control of progression through the different phases of the cell cycle by protein concentration. In [12], the authors consider in particular the influence of extracellular factors (nutrients) in the regulation of cell cycle duration in budding yeast. These nutrients are consumed by cells, and this triggers the progression through the cell cycle phases. Considering that nutrient consumption causes progression through cell cycle likely leads to delay differential equations of threshold type (see Kuang [38, Chapter 5.4] or Smith and Kuang [57]) that are close to state-dependent delay equations. Another approach consists in avoiding introduction of new variables (nutrient concentration, for instance) and describing cell cycle duration control by means of cell population counts.

A natural way of doing this is to consider that cell cycle durations depend either on the total population of HSCs or on the population of proliferating HSCs. Yet, proliferating cells are indeed cells committed to divide (once they have passed the $\mathrm{G}_{1} / \mathrm{S}$ transition) and consequently one can assume that they no longer consume nutrients. Hence, they would have no action on factors responsible for durations of cell cycles. On the contrary, the total population of HSCs, that is, both proliferating and nonproliferating cells, seems to be appropriate for controlling cell cycle lengths. However, proliferating HSCs represent only about $5 \%$ of all HSCs [18], so their role in cell cycle regulation can be neglected, and we will assume in this work, for simplicity, that only nonproliferating cells control cell cycle durations. The control of cell cycle durations by the total number of HSCs, although more natural and certainly appropriate, will not be considered in this work. This assumption is comforted by recent works [20,21] in which a modified version of Mackey's model [43], in which the total number of HSCs negatively controls HSC proliferation (in Mackey's model [43], only nonprolif- 
erating cells act on HSC proliferation), was studied, and both models were found to be qualitatively and quantitatively similar. Assuming the number of nonproliferating cells influences cell cycle durations modifies the nature of the time delay in Mackey's model [43] and transforms his system into a state-dependent delay system.

The theory of state-dependent delay differential equations has attracted a lot of attention over the last twenty years and started earlier in the 1960s. Driver [24], in 1963, studied existence of solutions, uniqueness, and continuous dependence on initial conditions for state-dependent delay differential equations, and his work has been developed and completed by various authors [9, 10, 37, 39, 48, 50, 51, 54, 59] while investigating periodic solutions for state-dependent delay equations. Nonlinear semigroups for some class of equations have been analyzed by Louihi, Hbid, and Arino [41]. The linearization of state-dependent delay equations has been studied by Cooke and Huang [19], Arino and Sanchez [11], Hartung [30], Hartung and Turi [31], Hartung et al. [32], and Walther [60]. A Hopf bifurcation theorem for state-dependent delay differential equations has been recently obtained by Eichmann [26]. Numerical methods for solving state-dependent delay differential equations have been investigated by Hofer, Tibken, and Lehn [35], Shampine [56], and the references therein. Despite the difficulty related to the analysis of state-dependent delay differential equations, they have been used in some applied works - in cell biology, for instance, by Bélair [13], Bélair, Mackey, and Mahaffy [14], Hbid, Sanchez, and Bravo de la Parra [34], Hofer, Tibken, and Lehn [36], and Mahaffy, Bélair, and Mackey [49], but also in automatic [61].

We propose a complete stability analysis of a nonlinear model of HSC dynamics, with state-dependent delay. The next section is devoted to the presentation of the model, which originally takes the form of an age-structured system. This system is reduced to a system of state-dependent delay equations in section 3. Properties of the resulting system, such as positivity and existence of steady states, are established in section 4 . We then focus on the stability of the trivial steady state and prove a sufficient condition for its global asymptotic stability using a Lyapunov-Razumikhin function in section 5. Next, we linearize the system about the unique positive steady state, which is shown to appear through a transcritical bifurcation of the trivial steady state, and perform the analysis of the Hopf bifurcation in section 6. A detailed numerical analysis, in section 7 , allows us to identify the role of each parameter and the influence of the state-dependent delay in stability switch of the positive steady state.

2. The model of HSC dynamics. Let us consider a population of HSCs, divided into two compartments: proliferating and resting (nonproliferating) cells. Denote by $n(t, a)$ and $p(t, a)$ the population densities of resting and proliferating cells, respectively, which have spent a time $a \geq 0$ in their phase at time $t \geq 0$.

As soon as a cell enters the proliferating phase, it is committed to divide a time $\tau$ later. We assume that the duration of the proliferating phase depends on the total population of nonproliferating cells

$$
N(t)=\int_{0}^{\infty} n(t, a) d a
$$

that is, $\tau=\tau(N(t))$. This choice (considering cell cycle durations are controlled only by nonproliferating cells) is justified above, in the introduction (paragraphs 5 and 6 ).

In [12], nutrients are considered as cell cycle duration regulators. These nutrients bind to cells and modify intracellular protein concentrations that decide on progres- 
sion through cell cycle phases. Assuming a constant concentration of nutrients, the cell cycle duration appears as a decreasing function of the available quantity of nutrients for each nonproliferating cell $[12,55]$ and consequently as an increasing function of $N(t)$. Hence the function $\tau$ is supposed to be bounded, positive, continuously differentiable, and increasing (a lack of HSCs is supposed to shorten cell cycles). We define

$$
\tau_{0}:=\inf _{x \geq 0} \tau(x)=\tau(0) \quad \text { and } \quad \tau_{\max }=\sup _{x \geq 0} \tau(x) .
$$

Proliferating cells can be lost by apoptosis (a programmed cell death) at a rate $\gamma \geq 0$, and, at mitosis, cells with age $\tau$ divide into two daughter cells which immediately enter the resting phase.

Nonproliferating cells are assumed to differentiate at a constant rate $\delta>0$, which can also take some natural mortality into account. They are introduced in the proliferating phase with a rate $\beta$, which is supposed to depend on the total population of nonproliferating cells (see Mackey [43, 44], Mackey and Rudnicki [46, 47], or PujoMenjouet et al. $[52,53]$ ), given by $(2.1)$. The function $\beta$ is supposed to be continuously differentiable, bounded, and positive. Furthermore, from a reasonable biological point of view, we assume $\beta$ is decreasing with $\lim _{x \rightarrow+\infty} \beta(x)=0$. Usually, it is believed that the function $\beta$ is a monotone decreasing Hill function (see [43]), given by

$$
\beta(x)=\frac{\beta_{0} \theta^{r}}{\theta^{r}+x^{r}}, \quad x \geq 0,
$$

with $\beta_{0}>0, \theta \geq 0$, and $r>0$. The parameter $\beta_{0}$ is the maximal rate of reentry in the proliferating phase, $\theta$ is the number of resting cells for which $\beta$ has its maximum rate of change with respect to the resting phase population, and $r$ describes the sensitivity of the reintroduction rate with changes in the population.

The evolution of HSC dynamics is described by the following system of agestructured partial differential equations:

$$
\begin{array}{llrl}
\frac{\partial n}{\partial t}+\frac{\partial n}{\partial a} & =-(\delta+\beta(N(t))) n, & & a>0, t>0, \\
\frac{\partial p}{\partial t}+\frac{\partial p}{\partial a} & =-\gamma p, & & 0<a<\tau(N(t)), t>0,
\end{array}
$$

where $N(t)$ denotes the total population of resting cells, defined by (2.1).

System (2.3)-(2.4) is completed by boundary conditions and initial conditions. The first ones describe the flux of cells entering each phase: new proliferating cells are nonproliferating cells introduced with a rate $\beta$, and new resting cells come from the division of proliferating cells that have spent a time $\tau(N(t))$ in the proliferating phase. Then the boundary conditions of (2.3)-(2.4) are

$$
\begin{aligned}
& n(t, 0)=2 p(t, \tau(N(t))), \\
& p(t, 0)=\beta(N(t)) N(t) .
\end{aligned}
$$

Moreover, we suppose that $\lim _{a \rightarrow+\infty} n(t, a)=0$.

Initial conditions of (2.3)-(2.4) are given by nonnegative $L^{1}$ functions $n_{0}$ and $p_{0}$ such that

$$
n(0, a)=n_{0}(a), a \geq 0, \quad \text { and } \quad p(0, a)=p_{0}(a), a \in[0, \tau(N(0))] .
$$

We are now going to check that system (2.3)-(2.4) reduces to a system of differential equations with state-dependent delay. 
3. Reduction to a state-dependent delay equation. First, we see that using the method of characteristics (see Webb [62]), the solution $p(t, a)$ of $(2.4),(2.6)$, and (2.7) is given, for $t>0$ and $0<a<\tau(N(t))$, by

$$
p(t, a)= \begin{cases}p_{0}(a-t) e^{-\gamma t} & \text { if } 0 \leq t<a, \\ \beta(N(t-a)) N(t-a) e^{-\gamma a} & \text { if } 0 \leq a \leq t .\end{cases}
$$

Since resting cells are introduced in the proliferating phase with a rate $\beta$, then $p_{0}(0)$, the population of cells introduced at time $t=0$ in the cycle, should satisfy the classical compatibility condition

$$
p_{0}(0)=\beta(N(0)) N(0) .
$$

This condition ensures the continuity of $p(t, a)$ on the line $t=a$.

Denote by $P(t)$ the total population of proliferating stem cells,

$$
P(t)=\int_{0}^{\tau(N(t))} p(t, a) d a, \quad t \geq 0 .
$$

Then, using (3.1), we deduce

$$
P(t)= \begin{cases}e^{-\gamma t}\left(\int_{0}^{t} e^{\gamma \theta} \beta(N(\theta)) N(\theta) d \theta+\int_{0}^{\tau(N(t))-t} p_{0}(\theta) d \theta\right), & t<\tau(N(t)), \\ e^{-\gamma t} \int_{t-\tau(N(t))}^{t} e^{\gamma \theta} \beta(N(\theta)) N(\theta) d \theta, & t \geq \tau(N(t)) .\end{cases}
$$

The variable $P(t)$ is straightforwardly continuous for $t \geq 0$, and since $\tau$ is continuously differentiable, $P(t)$ is also continuously differentiable provided that (3.2) holds true.

Integrating (2.3) with respect to the age variable, and using (2.5), we obtain

$$
N^{\prime}(t)=-\delta N(t)-\beta(N(t)) N(t)+2 p(t, \tau(N(t))) \quad \text { for } t \geq 0 .
$$

Using (3.1), we finally obtain, for $0 \leq t \leq \tau(N(t))$,

$$
N^{\prime}(t)=-\delta N(t)-\beta(N(t)) N(t)+2 p_{0}(\tau(N(t))-t) e^{-\gamma t},
$$

and, for $t>\tau(N(t))$,

$$
N^{\prime}(t)=-\delta N(t)-\beta(N(t)) N(t)+2 e^{-\gamma \tau(N(t))} \beta(N(t-\tau(N(t)))) N(t-\tau(N(t))) .
$$

Moreover, $N(0)=\int_{0}^{\infty} n_{0}(a) d a$.

One can notice that problem (3.4)-(3.5) is a nonlinear nonautonomous ordinary differential equation for $t<\tau(N(t))$, whereas, for $t \geq \tau(N(t))$, it is a system of differential equations with state-dependent delay. Let us concentrate on system (3.4)(3.5) for $t \in\left[0, \tau_{\max }\right]$.

Consider the differential equation

$$
\begin{aligned}
\frac{d x}{d t}(t) & =\left\{\begin{array}{l}
-\delta x(t)-\beta(x(t)) x(t)+2 p_{0}(\tau(x(t))-t) e^{-\gamma t}, \quad 0 \leq t \leq \tau(x(t)), \\
-\delta x(t)-\beta(x(t)) x(t) \\
+2 \beta(x(t-\tau(x(t)))) x(t-\tau(x(t))) e^{-\gamma \tau(x(t))}, \tau(x(t))<t \leq \tau_{\max },
\end{array}\right. \\
x(0) & =\int_{0}^{\infty} n_{0}(a) d a .
\end{aligned}
$$


For a smooth enough function $p_{0}$ satisfying (3.2), (3.6) has a unique continuously differentiable solution $\phi$ defined on $\left[0, \tau_{\max }\right]$ (see Walther [60]). Then (3.5) becomes the following delay differential equation for $t \geq \tau_{\max }$ :

$$
\left\{\begin{aligned}
\frac{d y}{d t}(t) & =-\delta y(t)-\beta(y(t)) y(t)+2 \beta(y(t-\tau(y(t)))) y(t-\tau(y(t))) e^{-\gamma \tau(y(t))}, \\
y(\theta) & =\phi(\theta), \quad 0 \leq \theta \leq \tau_{\max } .
\end{aligned}\right.
$$

Thus, by making a change of variable $N(t):=y\left(t+\tau_{\max }\right)$, system (3.4)-(3.5) can be written as a state-dependent delay differential equation for $t \geq 0$,

$$
N^{\prime}(t)=-(\delta+\beta(N(t))) N(t)+2 e^{-\gamma \tau(N(t))} \beta(N(t-\tau(N(t)))) N(t-\tau(N(t))),
$$

with, for $\theta \in\left[-\tau_{\max }, 0\right], N(\theta)=\phi\left(\theta+\tau_{\max }\right)$.

Existence and uniqueness of solutions of (3.7) cannot be easily deduced. Equation (3.7) writes $x^{\prime}(t)=g(x(t), x(t-\tau(x(t))))$ for $t \geq 0$, where the function $g:\left(\mathbb{R}^{+}\right)^{2} \rightarrow \mathbb{R}$ is given by

$$
g(x, y)=-(\delta+\beta(x)) x+2 e^{-\gamma \tau(x)} \beta(y) y, \quad(x, y) \in\left(\mathbb{R}^{+}\right)^{2} .
$$

This equation can also be written in the general form

$$
x^{\prime}(t)=f\left(x_{t}\right) \quad \text { for } t \geq 0,
$$

where $x_{t}$ is defined by $x_{t}(\theta)=x(t+\theta)$ for $\theta \in\left[-\tau_{\max }, 0\right]$, and the function $f: C \rightarrow \mathbb{R}$ is given, for $\phi \in C$, the space of continuous functions on $\left[-\tau_{\max }, 0\right]$, by

$$
f(\phi)=g(\phi(0), \phi(-\tau(\phi(0)))) .
$$

Since the functions $\beta(x)$ and $\tau(x)$ are continuously differentiable on $[0,+\infty)$, then $g$ is also continuously differentiable on $\left(\mathbb{R}^{+}\right)^{2}$. Therefore, existence and uniqueness of a solution of (3.7) defined on $[0,+\infty)$ for an initial condition belonging to $C^{1}$, the space of continuously differentiable functions on $\left[-\tau_{\max }, 0\right]$, follow from MalletParet, Nussbaum, and Paraskevopoulos [50]. One may note that it is not reasonable to expect a well-posed state-dependent delay differential problem from searching for solutions in $C$ (see Walther [60]).

One can notice that (3.7) does not depend on the proliferating cell population $P$, whereas the converse is not true.

In the next section, we focus on some properties of (3.7), such as positivity and boundedness of solutions, as well as the existence of steady states.

4. Properties of the model and existence of steady states. In this section we focus on basic properties of solutions of (3.7), such as positivity and boundedness. We also concentrate on the existence of steady states. We suppose from now on that existence and uniqueness of solutions of (3.7) hold for $t \in\left[-\tau_{\max },+\infty\right)$.

The solutions of (3.7) represent cell numbers; therefore, it is essential to show positivity and boundedness. Such properties are straightforwardly obtained (for instance, using a method similar to the one presented in Adimy, Crauste, and Ruan [5]). They are stated in the following proposition.

Proposition 4.1. The solutions of (3.7) are nonnegative, and, provided that $\delta>0$, they are bounded.

The expression of $P(t)$ in (3.3) gives more precise information on the influence of the behavior of $N(t)$ on the stability and periodicity of the solutions $P(t)$. The following lemma, which states these results, is immediately obtained by using (3.3). 
Lemma 4.2. Let $(P(t), N(t))$ be a solution of (3.3) and (3.7). If $N_{\infty}:=$ $\lim _{t \rightarrow \infty} N(t)$ exists, then

$$
\lim _{t \rightarrow \infty} P(t)= \begin{cases}N_{\infty} \beta\left(N_{\infty}\right)\left(\frac{1-e^{-\gamma \tau\left(N_{\infty}\right)}}{\gamma}\right) & \text { if } \gamma>0, \\ N_{\infty} \beta\left(N_{\infty}\right) \tau\left(N_{\infty}\right) & \text { if } \gamma=0 .\end{cases}
$$

If $N(t)$ is T-periodic, then $P(t)$ is also T-periodic.

In particular, Lemma 4.2 shows the influence of (3.7) on the stability of the entire system. We now focus on the existence of steady states of (3.7).

Proposition 4.3. Assume $\delta>0$ and

$$
\tau_{0}<\frac{1}{\gamma} \ln \left(\frac{2 \beta(0)}{\beta(0)+\delta}\right) .
$$

Then equation (3.7) has two steady states $N \equiv 0$ and $N \equiv N^{*}>0$, where

$$
\left(2 e^{-\gamma \tau\left(N^{*}\right)}-1\right) \beta\left(N^{*}\right)=\delta .
$$

If (4.1) does not hold, then $N \equiv 0$ is the only steady state of (3.7).

Proof. Define, for $N \geq 0$, the function $\chi(N):=\left(2 e^{-\gamma \tau(N)}-1\right) \beta(N)$. Then

$$
\chi(0)=\left(2 e^{-\gamma \tau_{0}}-1\right) \beta(0)>\delta \quad \text { and } \quad \lim _{N \rightarrow+\infty} \chi(N)=0 .
$$

Consequently, (4.2) has at least one positive solution $N^{*}$. Moreover, since $\tau$ is increasing, then $\chi$ is decreasing, and, provided that $\chi(N)>0, N^{*}$ is unique.

In the next section, we concentrate on the asymptotic stability of the trivial steady state of (3.7).

5. Asymptotic stability of the trivial steady state. In the next theorem, we give a necessary and sufficient condition for the trivial steady state of (3.7), when it is unique, to be globally asymptotically stable using a Lyapunov-Razumikhin function presented in [29].

We recall that $\delta>0$. From Proposition 4.1 all solutions of (3.7) are then bounded.

Theorem 5.1. Assume

$$
\frac{1}{\gamma} \ln \left(\frac{2 \beta(0)}{\delta}\right)<\tau_{0} .
$$

Then the trivial steady state of (3.7) is globally asymptotically stable.

Proof. Consider the Lyapunov function $V: \mathbb{R}^{+} \rightarrow \mathbb{R}^{+}$given by $V(x)=x^{2} / 2$. We have, for $x \in \mathbb{R}^{+}, u(x) \leq V(x) \leq v(x)$, with $u(x)=x^{2} / 2$ and $v(x)=x^{2}$.

Define $p: \mathbb{R}^{+} \rightarrow \mathbb{R}^{+}$by $p(x)=x e^{2 \alpha \tau(\sqrt{2 x})}, x \in \mathbb{R}^{+}$, with $0<\alpha<\min \{\gamma$, $\left.\gamma-\ln (2 \beta(0) / \delta) / \tau_{0}\right\}$. Let $N$ be a solution of (3.7) such that, for $t \geq 0, \theta \in\left[-\tau_{\max }, 0\right]$,

$$
V(N(t+\theta))<p(V(N(t))) .
$$

Then, for $t \geq 0, N(t-\tau(N(t))) \leq e^{\alpha \tau(N(t))} N(t)$. It follows that, for $t \geq 0$,

$$
\begin{aligned}
\dot{V}(N(t))= & -(\delta+\beta(N(t))) N(t)^{2} \\
& +2 e^{-\gamma \tau(N(t))} \beta(N(t-\tau(N(t)))) N(t-\tau(N(t))) N(t) \\
\leq & -\delta N(t)^{2}+2 e^{-\gamma \tau(N(t))} \beta(0) e^{\alpha \tau(N(t))} N(t)^{2} \\
= & -\left[\delta-2 e^{-(\gamma-\alpha) \tau(N(t))} \beta(0)\right] N(t)^{2} .
\end{aligned}
$$


Let $W: \mathbb{R}^{+} \rightarrow \mathbb{R}$ be the map defined, for $x \in \mathbb{R}^{+}$, by $W(x)=\left[\delta-2 e^{-(\gamma-\alpha) \tau(x)} \beta(0)\right] x^{2}$. From (5.1), $W$ is a positive nondecreasing function. Moreover, (5.3) gives $\dot{V}(N(t)) \leq$ $-W(N(t))$ whenever (5.2) holds true. Since $u(r) \rightarrow \infty$ as $r \rightarrow \infty$, conditions of [29, Theorem 5.2] hold, and so does the conclusion.

The global asymptotic stability of $N \equiv 0$ is obtained in Theorem 5.1 with an assumption, condition (5.1), that corresponds only to a part of the domain where $N \equiv 0$ is the only steady state of (3.7), contrary to what can be noted for a constant time delay (see, for instance, Adimy, Crauste, and Ruan [5]). We can, however, complete this result by studying the local asymptotic stability of the steady states.

Next, we analyze the local asymptotic stability of the two steady states of system (3.7), $N \equiv 0$ and $N \equiv N^{*}$, by studying the sign of the real parts of eigenvalues of the associated characteristic equations (see [32,60] for more details about linearization and stability of state-dependent delay differential equations).

Let $J: C^{1} \rightarrow \mathbb{R}$ be the map defined, for $\phi \in C^{1}$, by $J(\phi)=\phi(-\tau(\phi(0)))$. The derivatives of $J$ have the form

$$
\frac{d}{d \phi} J(\phi) \psi=-\tau^{\prime}(\phi(0)) \phi^{\prime}(-\tau(\phi(0))) \psi(0)+\psi(-\tau(\phi(0))), \quad \phi, \psi \in C^{1} .
$$

Then, for a steady state $x^{*} \in\left\{0, N^{*}\right\}$ of (3.7), the linearized function $f$ of (3.8) around $x^{*}$ is, for $\psi \in C^{1}$,

$$
\begin{aligned}
D f\left(x^{*}\right) \psi= & -\left[\delta+\beta\left(x^{*}\right)+\beta^{\prime}\left(x^{*}\right) x^{*}\right] \psi(0)-2 \gamma e^{-\gamma \tau\left(x^{*}\right)} \tau^{\prime}\left(x^{*}\right) \beta\left(x^{*}\right) x^{*} \psi(0) \\
& +2 e^{-\gamma \tau\left(x^{*}\right)}\left[\beta^{\prime}\left(x^{*}\right) x^{*}+\beta\left(x^{*}\right)\right] \psi\left(-\tau\left(x^{*}\right)\right) .
\end{aligned}
$$

It follows that the characteristic equation associated with the linearization of (3.7) around $x^{*}$ is

$$
\Delta(\lambda)=\lambda+\delta+\bar{\beta}+\tau^{\prime}\left(x^{*}\right) \bar{\alpha} e^{-\gamma \tau\left(x^{*}\right)}-2 \bar{\beta} e^{-\gamma \tau\left(x^{*}\right)} e^{-\lambda \tau\left(x^{*}\right)},
$$

where

$$
\bar{\alpha}=2 \gamma \beta\left(x^{*}\right) x^{*}, \quad \bar{\beta}=\beta\left(x^{*}\right)+\beta^{\prime}\left(x^{*}\right) x^{*} .
$$

We easily obtain the following theorem.

THEOREM 5.2. The trivial steady state of (3.7) is unstable when (4.1) holds true and locally asymptotically stable when (4.1) does not hold.

Proof. The characteristic equation (5.4), when $x^{*}=0$, is given by

$$
\Delta(\lambda)=\lambda+\delta+\beta(0)-2 \beta(0) e^{-\gamma \tau_{0}} e^{-\lambda \tau_{0}} .
$$

Let $\lambda \in \mathbb{R}$. We have

$$
\begin{gathered}
\frac{d \Delta}{d \lambda}(\lambda)=1+2 \tau_{0} e^{-\gamma \tau_{0}} \beta(0) e^{-\lambda \tau_{0}}>0, \\
\Delta(0)=\delta+\beta(0)-2 \beta(0) e^{-\gamma \tau_{0}}=\delta-\left(2 e^{-\gamma \tau_{0}}-1\right) \beta(0),
\end{gathered}
$$

and $\lim _{\lambda \rightarrow \infty} \Delta(\lambda)=+\infty$. Then there exists $\lambda_{0} \in \mathbb{R}$, which is unique, such that $\Delta\left(\lambda_{0}\right)=0$. When (4.1) holds, then $\Delta(0)<0$, so $\lambda_{0}>0$, which proves the instability of the trivial steady state.

Conversely, when (4.1) does not hold, $\Delta(0) \geq 0$ and $\lambda_{0} \leq 0$. One can show (see [20], for instance) that all roots $\lambda \neq \lambda_{0}$ of $\Delta$ satisfy $\operatorname{Re}(\lambda)<\lambda_{0}$. Consequently, the local asymptotic stability straightforwardly follows when (4.1) does not hold. 
From Theorems 5.1 and 5.2 , we obtain that the trivial steady state $N \equiv 0$ of (3.7) is globally asymptotically stable when

$$
\frac{1}{\gamma} \ln \left(\frac{2 \beta(0)}{\delta}\right)<\tau_{0},
$$

locally asymptotically stable when

$$
\frac{1}{\gamma} \ln \left(\frac{2 \beta(0)}{\beta(0)+\delta}\right)<\tau_{0} \leq \frac{1}{\gamma} \ln \left(\frac{2 \beta(0)}{\delta}\right),
$$

and unstable when

$$
\tau_{0}<\frac{1}{\gamma} \ln \left(\frac{2 \beta(0)}{\beta(0)+\delta}\right) .
$$

In the next section we focus on the local asymptotic stability of the steady state $N^{*}$ of (3.7). In particular, we study the existence of a Hopf bifurcation that would destabilize the steady state and create periodic solutions.

6. Transcritical bifurcation, Hopf bifurcation, and periodic solutions. This section is devoted to the local asymptotic stability analysis of the positive steady state $N \equiv N^{*}$ of (3.7). We are going to investigate the sign of real parts of eigenvalues of (5.4) to obtain the existence of a local Hopf bifurcation (see [26]).

Throughout this section, we assume the function $\tau$ is given by $\tau(x)=\mu \tilde{\tau}(x)$, where $\mu$ is a positive parameter and $\tilde{\tau}: \mathbb{R}^{+} \rightarrow \mathbb{R}^{+}$is a positive, increasing, bounded, and differentiable function. Equation (3.7) reads

$$
N^{\prime}(t)=-(\delta+\beta(N(t))) N(t)+2 e^{-\gamma \mu \tilde{\tau}(N(t))} \beta(N(t-\mu \tilde{\tau}(N(t)))) N(t-\mu \tilde{\tau}(N(t))) .
$$

Moreover, we assume that condition (4.1) holds to ensure the existence of the positive steady state $N^{*}$ of (3.7). Condition (4.1) is equivalent to

$$
\beta(0)>\delta \quad \text { and } \quad 0 \leq \mu<\frac{1}{\tilde{\tau}(0) \gamma} \ln \left(\frac{2 \beta(0)}{\delta+\beta(0)}\right):=\bar{\mu} .
$$

This describes the fact that the maximal introduction rate $\beta(0)$ has to be larger than the mortality rate $\delta$, and the cell cycle duration $\tau$ cannot be too long for system (6.1) to exhibit a steady state other than the one describing the cell's dying out.

The positive steady state $N^{*}$ depends then on the parameter $\mu$ and is given implicitly by

$$
\left(2 e^{-\gamma \mu \tilde{\tau}\left(N^{*}(\mu)\right)}-1\right) \beta\left(N^{*}(\mu)\right)=\delta, \quad \mu \in[0, \bar{\mu}) .
$$

Thus, by using the implicit function theorem, $N^{*}$ is a decreasing continuously differentiable function of $\mu$. Furthermore, using (6.2) and (6.3), one obtains

$$
N^{*}(\mu=0)=\beta^{-1}(\delta) \quad \text { and } \quad \lim _{\mu \rightarrow \bar{\mu}} N^{*}(\mu)=0 .
$$

Taking $\mu$ as a parameter, our purpose is to prove the existence of the local Hopf bifurcation of (6.1). 
From (5.4), the characteristic equation associated with $N^{*}(\mu)$ is written as

$$
\begin{aligned}
\Delta(\lambda, \mu)= & \lambda+\delta+\bar{\beta}(\mu)+\mu \tilde{\tau}^{\prime}\left(N^{*}(\mu)\right) \bar{\alpha}(\mu) e^{-\gamma \mu \tilde{\tau}\left(N^{*}(\mu)\right)} \\
& -2 \bar{\beta}(\mu) e^{-\gamma \mu \tilde{\tau}\left(N^{*}(\mu)\right)} e^{-\lambda \mu \tilde{\tau}\left(N^{*}(\mu)\right)}
\end{aligned}
$$

where

$$
\bar{\alpha}(\mu)=2 \gamma \beta\left(N^{*}(\mu)\right) N^{*}(\mu) \quad \text { and } \quad \bar{\beta}(\mu)=\beta\left(N^{*}(\mu)\right)+\beta^{\prime}\left(N^{*}(\mu)\right) N^{*}(\mu) .
$$

The next result states the existence of a transcritical bifurcation of the positive steady state when $\mu=\bar{\mu}$.

THEOREM 6.1. When $\mu=\bar{\mu}$, the positive steady state undergoes a transcritical bifurcation, that is, for $\mu<\bar{\mu}, \mu$ close to $\bar{\mu}$, the positive steady state is locally asymptotically stable, whereas the trivial steady state is unstable, and for $\mu>\bar{\mu}$ the trivial steady state is locally asymptotically stable and is the only steady state of (6.1).

Proof. The stability of the trivial steady state follows from Theorem 5.2. We investigate local asymptotic stability of $N^{*}(\mu)$ in a neighborhood of $\bar{\mu}$.

First, notice that when $\bar{\beta}(\mu)>0$, characteristic roots of (6.5) have negative real parts. Indeed, assume $\bar{\beta}(\mu)>0$, and consider $\Delta(\lambda, \mu)$ as a function of real $\lambda$. Then $\lambda \mapsto \Delta(\lambda, \mu)$ is an increasing function such that $\lim _{\lambda \rightarrow+\infty} \Delta(\lambda, \mu)=+\infty$ and, using the above definition of $\bar{\beta}(\mu)$,

$\Delta(0, \mu)=-\left(2 e^{-\gamma \mu \widetilde{\tau}\left(N^{*}(\mu)\right)}-1\right) N^{*}(\mu) \beta^{\prime}\left(N^{*}(\mu)\right)+\mu \widetilde{\tau}^{\prime}\left(N^{*}(\mu)\right) \bar{\alpha}(\mu) e^{-\gamma \mu \widetilde{\tau}\left(N^{*}(\mu)\right)}>0$.

Thus, there exists a unique $\lambda^{*}<0$ such that $\Delta\left(\lambda^{*}, \mu\right)=0$. Separating real and imaginary parts in (6.5), one can easily show that all characteristic roots $\lambda \neq \lambda^{*}$ satisfy $\operatorname{Re}(\lambda)<\lambda^{*}$. The local asymptotic stability of the positive steady state when $\bar{\beta}(\mu)>0$ immediately follows.

Finally, one has to note that when $\mu$ is close to $\bar{\mu}, \mu<\bar{\mu}$, then $N^{*}(\mu)$ is close to zero (see (6.4)); therefore, $\bar{\beta}(\mu) \approx \beta(0)>0$. The conclusion follows.

Local asymptotic stability of the positive steady state when $\mu=0$ is established in the following lemma.

Lemma 6.2. Assume $\beta(0)>\delta$ and $\mu=0$. Then the steady state $N^{*}$ of system (6.1) is locally asymptotically stable.

Proof. Let $\mu=0$. From (6.5), $\Delta(\lambda, 0)=\lambda+\delta-\bar{\beta}(0)$. Then, $\lambda=\bar{\beta}(0)-\delta$ is the unique eigenvalue associated with the characteristic equation. On the other hand, $N^{*}(0)=\beta^{-1}(\delta)>0$, so $\lambda=\beta^{\prime}\left(N^{*}(0)\right) N^{*}(0)<0$. This concludes the proof.

Thus, $N^{*}(\mu)$ is locally asymptotically stable for $\mu=0$ and the stability can be lost as $\mu$ increases away from 0 , with $\mu<\bar{\mu}$, only if purely imaginary characteristic roots appear. One may note that $N^{*}(\mu)$ is locally asymptotically stable for $\mu<\bar{\mu}, \mu$ close to $\bar{\mu}$ (see Theorem 6.1), and so if stability is lost as $\mu$ increases away from zero, a second switch must be observed as $\mu$ keeps increasing and reaches $\bar{\mu}$.

Define, for $\mu \in[0, \bar{\mu})$,

$b(\mu)=\delta+\bar{\beta}(\mu)+\mu \tilde{\tau}^{\prime}\left(N^{*}(\mu)\right) \bar{\alpha}(\mu) e^{-\gamma \mu \tilde{\tau}\left(N^{*}(\mu)\right)} \quad$ and $\quad c(\mu)=-2 \bar{\beta}(\mu) e^{-\gamma \mu \tilde{\tau}\left(N^{*}(\mu)\right)}$.

Then (6.5) becomes

$$
\Delta(\lambda, \mu)=\lambda+b(\mu)+c(\mu) e^{-\lambda \mu \tilde{\tau}\left(N^{*}(\mu)\right)} .
$$


In the following, we investigate the existence of purely imaginary roots of (6.6). It is obvious that $\lambda=0$ is not a characteristic root of (6.6). Indeed,

$$
\begin{aligned}
b(\mu)+c(\mu)=\delta & +\beta\left(N^{*}(\mu)\right)\left(1-2 e^{-\gamma \mu \tilde{\tau}\left(N^{*}(\mu)\right)}\right) \\
& +\beta^{\prime}\left(N^{*}(\mu)\right)\left(1-2 e^{-\gamma \mu \tilde{\tau}\left(N^{*}(\mu)\right)}\right) N^{*}(\mu) \\
& +2 \gamma \mu \tilde{\tau}^{\prime}\left(N^{*}(\mu)\right) \beta\left(N^{*}(\mu)\right) N^{*}(\mu) e^{-\gamma \mu \tilde{\tau}\left(N^{*}(\mu)\right)} .
\end{aligned}
$$

Since $\delta-\left(2 e^{-\gamma \mu \tilde{\tau}\left(N^{*}(\mu)\right)}-1\right) \beta\left(N^{*}(\mu)\right)=0$, we obtain $1-2 e^{-\gamma \mu \tilde{\tau}\left(N^{*}(\mu)\right)}<0$ and

$$
\Delta(0, \mu)=b(\mu)+c(\mu)>0 .
$$

It follows that $\lambda=0$ is not an eigenvalue.

Let $\omega>0$. Separating real and imaginary parts, equality $\Delta(i \omega, \mu)=0$ is equivalent to

$$
\left\{\begin{aligned}
\omega & =c(\mu) \sin \left(\omega \mu \tilde{\tau}\left(N^{*}(\mu)\right)\right), \\
b(\mu) & =-c(\mu) \cos \left(\omega \mu \tilde{\tau}\left(N^{*}(\mu)\right)\right) .
\end{aligned}\right.
$$

One can note that if $i \omega$ is a purely imaginary root of (6.6) then so is $-i \omega$. A necessary condition for (6.6) to have purely imaginary roots is

$$
|c(\mu)|>|b(\mu)| \text {. }
$$

If no $\mu \in[0, \bar{\mu})$ fulfills condition (6.8), then the characteristic equation (6.6) has no purely imaginary root. Consequently, from Lemma 6.2, all eigenvalues of (6.6) have negative real parts and the steady state $N^{*}(\mu)$ is locally asymptotically stable for all $\mu \in[0, \bar{\mu})$.

We have already checked that $b(\mu)+c(\mu)>0$. Then, for (6.8) to hold true, it is necessary that $c(\mu)>0$, that is, $\bar{\beta}(\mu)<0$. A sufficient condition for $(6.8)$ is then $b(\mu)<0$, which is equivalent to

$$
\begin{aligned}
& \delta+\beta\left(N^{*}(\mu)\right)+\beta^{\prime}\left(N^{*}(\mu)\right) N^{*}(\mu) \\
& +2 \gamma \mu \tilde{\tau}^{\prime}\left(N^{*}(\mu)\right) \beta\left(N^{*}(\mu)\right) N^{*}(\mu) e^{-\gamma \mu \tilde{\tau}\left(N^{*}(\mu)\right)}<0 .
\end{aligned}
$$

We have the following lemma.

Lemma 6.3. Let $\eta:[0,+\infty) \rightarrow(-\infty, 0]$ and $\sigma:[0,+\infty) \rightarrow[0,+\infty)$ be defined, for $y \geq 0$, by $\eta(y)=y \beta^{\prime}(y)$ and $\sigma(y)=y \tilde{\tau}^{\prime}(y)$. Assume the following:

(R1) $\eta$ and $\sigma$ are decreasing on the interval $\left[0, \beta^{-1}(\delta)\right]$.

(R2) $\eta\left(\beta^{-1}(\delta)\right)<-2 \delta$.

Then there exists a unique $\mu^{*} \in(0, \bar{\mu})$ such that condition (6.9) is satisfied if and only if $\mu \in\left[0, \mu^{*}\right)$.

Proof. Let $h_{1}$ and $h_{2}$ be the negative functions defined, for $\mu \in[0, \bar{\mu})$, by

$$
h_{1}(\mu)=\eta\left(N^{*}(\mu)\right) \quad \text { and } \quad h_{2}(\mu)=-\left(\delta+\beta\left(N^{*}(\mu)\right)\right)\left(1+2 \gamma \mu \sigma\left(N^{*}(\mu)\right)\right) .
$$

Then, from (6.3), $\{\mu \in[0, \bar{\mu}):$ condition (6.9) is satisfied $\}=\left\{\mu \in[0, \bar{\mu}): h_{1}(\mu)<\right.$ $\left.h_{2}(\mu)\right\}$. Since $\beta$ is decreasing on $[0,+\infty), N^{*}$ is decreasing on $[0, \bar{\mu})$, and, from (R1), $\eta$ and $\sigma$ are decreasing on $\left[0, \beta^{-1}(\delta)\right]$, we deduce that $h_{1}$ is increasing and $h_{2}$ is decreasing. Moreover, from (6.4), $\lim _{\mu \rightarrow \bar{\mu}} N^{*}(\mu)=0$. It follows that

$$
\begin{array}{ll}
h_{1}(0)=\eta\left(\beta^{-1}(\delta)\right)<0, & \lim _{\mu \rightarrow \bar{\mu}} h_{1}(\mu)=\eta(0)=0, \\
h_{2}(0)=-2 \delta, & \lim _{\mu \rightarrow \bar{\mu}} h_{2}(\mu)=-(\delta+\beta(0)) .
\end{array}
$$


From (R2), it follows that $h_{1}(0)<h_{2}(0)$. Consequently there exists a unique $\mu^{*} \in$ $(0, \bar{\mu})$ such that $\left\{\mu \in[0, \bar{\mu}): h_{1}(\mu)<h_{2}(\mu)\right\}=\left[0, \mu^{*}\right)$. This achieves the proof.

In what follows, we assume there exists $\mu^{*} \in(0, \bar{\mu})$ such that (6.8) is fulfilled for $\mu \in\left[0, \mu^{*}\right)$.

System (6.7) is equivalent to

$$
\cos \left(\omega \mu \tau^{*}(\mu)\right)=-\frac{b(\mu)}{c(\mu)}, \quad \sin \left(\omega \mu \tau^{*}(\mu)\right)=\frac{\omega}{c(\mu)},
$$

where $\tau^{*}(\mu)=\tilde{\tau}\left(N^{*}(\mu)\right)$. Therefore, adding the squares of both sides of (6.10), purely imaginary eigenvalues $i \omega$ of (6.6), with $\omega>0$, must satisfy

$$
\omega=\left(c^{2}(\mu)-b^{2}(\mu)\right)^{\frac{1}{2}} .
$$

Thus, in the following, we will think of $\omega$ as $\omega(\mu), \mu \in\left[0, \mu^{*}\right)$. Substituting expression (6.11) for $\omega$ in (6.10), we obtain

$$
\left\{\begin{aligned}
\cos \left(\mu \tau^{*}(\mu)\left(c^{2}(\mu)-b^{2}(\mu)\right)^{\frac{1}{2}}\right) & =-\frac{b(\mu)}{c(\mu)} \\
\sin \left(\mu \tau^{*}(\mu)\left(c^{2}(\mu)-b^{2}(\mu)\right)^{\frac{1}{2}}\right) & =\frac{\left(c^{2}(\mu)-b^{2}(\mu)\right)^{\frac{1}{2}}}{c(\mu)}
\end{aligned}\right.
$$

From the above reasoning, values of $\mu \in\left[0, \mu^{*}\right)$ solutions of system (6.12) generate positive $\omega(\mu)$ given by (6.11) and hence yield imaginary eigenvalues of (6.6). Consequently, we look for positive solutions $\mu$ of (6.12) in the interval $\left[0, \mu^{*}\right)$. They satisfy

$$
\mu \tau^{*}(\mu)\left(c^{2}(\mu)-b^{2}(\mu)\right)^{\frac{1}{2}}=\arccos \left(-\frac{b(\mu)}{c(\mu)}\right)+2 k \pi, \quad k \in \mathbb{N}_{0},
$$

where $\mathbb{N}_{0}$ denotes the set of all nonnegative integers. We set

$$
\mu^{k}(\mu):=\frac{\arccos \left(-\frac{b(\mu)}{c(\mu)}\right)+2 k \pi}{\tau^{*}(\mu)\left(c^{2}(\mu)-b^{2}(\mu)\right)^{\frac{1}{2}}}, \quad k \in \mathbb{N}_{0}, \mu \in\left[0, \mu^{*}\right) .
$$

Values of $\mu$ for which $\omega(\mu)=\left(c^{2}(\mu)-b^{2}(\mu)\right)^{\frac{1}{2}}$ is a solution of $(6.7)$ are roots of the functions

$$
Z_{k}(\mu)=\mu-\mu^{k}(\mu), \quad k \in \mathbb{N}_{0}, \mu \in\left[0, \mu^{*}\right) .
$$

The roots of $Z_{k}$ can be found using popular software but are hard to determine with analytical tools [15]. The following lemma states some straightforward properties of the $Z_{k}$ functions.

Lemma 6.4. For $k \in \mathbb{N}_{0}$,

$$
Z_{k}(0)<0 \quad \text { and } \quad \lim _{\mu \rightarrow \mu^{*}} Z_{k}(\mu)=-\infty .
$$

Therefore, provided that no root of $Z_{k}$ is a local extremum, the number of positive roots of $Z_{k}, k \in \mathbb{N}_{0}$, on the interval $\left[0, \mu^{*}\right)$ is even. Moreover, if $Z_{k}$ has no root on the interval $\left[0, \mu^{*}\right)$, then $Z_{j}$, with $j>k$, does not have positive roots. 
Remark 1. The last statement in Lemma 6.4 implies, in particular, that, if $Z_{0}$ has no positive root, then (6.7) has no positive solution, and (6.6) does not have purely imaginary roots.

The search for purely imaginary roots ends up finding positive real roots of real functions $Z_{k}$ that can mostly be handled numerically. In the following proposition, however, we establish some properties of purely imaginary roots of (6.6) using a method described in [38].

Proposition 6.5. Let $\pm i \omega\left(\mu_{c}\right)$, with $\omega\left(\mu_{c}\right)>0$ and $\mu_{c} \in\left(0, \mu^{*}\right)$, be a pair of purely imaginary roots of (6.6) when $\mu=\mu_{c}$. Then $\pm i \omega\left(\mu_{c}\right)$ are simple roots of (6.6) such that

$$
\begin{aligned}
& \operatorname{sign}\left\{\frac{d \mathcal{R} e(\lambda(\mu))}{d \mu}\right\}_{\mu=\mu_{c}} \\
& =\operatorname{sign}\left\{c^{3}\left(\mu_{c} \hat{\tau}_{c}^{\prime}+\tau_{c}^{*}\right)+c^{2} c^{\prime} \mu_{c} \tau^{*}-c\left(b^{2}\left(\mu_{c} \hat{\tau}_{c}^{\prime}+\tau_{c}^{*}\right)+b^{\prime}+b b^{\prime} \mu_{c} \tau_{c}^{*}\right)+c^{\prime} b\right\},
\end{aligned}
$$

with $\tau_{c}^{*}=\tau^{*}\left(\mu_{c}\right), \hat{\tau}_{c}^{\prime}=d \tau^{*}\left(\mu_{c}\right) / d \mu, b=b\left(\mu_{c}\right), c=c\left(\mu_{c}\right), b^{\prime}=b^{\prime}\left(\mu_{c}\right)$, and $c^{\prime}=c^{\prime}\left(\mu_{c}\right)$.

Proof. Let $\lambda(\mu)$ be a branch of roots of $(6.6)$, so $\Delta(\lambda(\mu), \mu)=0$, such that $\lambda\left(\mu_{c}\right)=i \omega\left(\mu_{c}\right)$. Then,

$$
\frac{d \lambda}{d \mu}(\mu) \Delta_{\lambda}(\lambda, \mu)+\Delta_{\mu}(\lambda, \mu)=0
$$

where

$$
\Delta_{\lambda}(\lambda, \mu):=\frac{d \Delta}{d \lambda}(\lambda, \mu)=1-c(\mu) \mu \tau^{*}(\mu) e^{-\lambda \mu \tau^{*}(\mu)},
$$

and

$$
\Delta_{\mu}(\lambda, \mu):=\frac{d \Delta}{d \mu}(\lambda, \mu)=b^{\prime}(\mu)-\left[c(\mu)\left(\mu \hat{\tau}^{\prime}(\mu)+\tau^{*}(\mu)\right) \lambda-c^{\prime}(\mu)\right] e^{-\lambda \mu \tau^{*}(\mu)},
$$

with $\hat{\tau}^{\prime}(\mu)=d \tilde{\tau}\left(N^{*}(\mu)\right) / d \mu$.

Assume, by contradiction, that $\lambda\left(\mu_{c}\right)=i \omega\left(\mu_{c}\right)$ is not a simple root of (6.6). Then

$$
\Delta_{\lambda}\left(i \omega\left(\mu_{c}\right), \mu_{c}\right)=1-\mu_{c} \tau_{c}^{*} c\left(\mu_{c}\right) e^{-i \omega\left(\mu_{c}\right) \mu_{c} \tau_{c}^{*}}=0 .
$$

Using (6.6) for $\mu=\mu_{c}$, we then deduce $b\left(\mu_{c}\right)+1 /\left(\mu_{c} \tau_{c}^{*}\right)+i \omega\left(\mu_{c}\right)=0$, and consequently $\omega\left(\mu_{c}\right)=0$, which gives a contradiction. It follows that all purely imaginary roots of (6.6) are simple.

In the following, we do not mention the dependence of the coefficients $\tau^{*}, \hat{\tau}^{\prime}, b$, and $c$ (and their derivatives) with respect to $\mu$.

Since $\Delta(\lambda, \mu)=0$, we deduce $e^{\lambda \mu \tau^{*}}=-c /(\lambda+b)$. Therefore, from (6.15) we obtain

$$
\left(\frac{d \lambda}{d \mu}\right)^{-1}=\frac{c+c \mu \tau^{*}(\lambda+b)}{\left[c^{\prime}-c \lambda\left(\mu \hat{\tau}^{\prime}+\tau^{*}\right)\right](\lambda+b)-b^{\prime} c} .
$$

Taking the real part of the above equality for $\mu=\mu_{c}$, we obtain

$$
\begin{aligned}
& \left.\mathcal{R} e\left(\frac{d \lambda}{d \mu}\right)^{-1}\right|_{\mu=\mu_{c}} \\
& =\frac{\left[c^{2}\left(1+b \mu_{c} \tau_{c}^{*}\right)\left(\mu_{c} \hat{\tau}_{c}^{\prime}+\tau_{c}^{*}\right)+\mu_{c} \tau_{c}^{*} c\left(c^{\prime}-b c\left(\mu_{c} \hat{\tau}_{c}^{\prime}+\tau_{c}^{*}\right)\right)\right] \omega\left(\mu_{c}\right)^{2}+c\left(1+b \mu_{c} \tau_{c}^{*}\right)\left(c^{\prime} b-b^{\prime} c\right)}{\left(b^{\prime} c-c^{\prime} b-c\left(\mu_{c} \hat{\tau}_{c}^{\prime}+\tau_{c}^{*}\right) \omega\left(\mu_{c}\right)^{2}\right)^{2}+\left(c^{\prime}-b c\left(\mu_{c} \hat{\tau}_{c}^{\prime}+\tau_{c}^{*}\right)\right) \omega\left(\mu_{c}\right)^{2}} .
\end{aligned}
$$


Notice that $\operatorname{sign}\{d \mathcal{R} e(\lambda) / d \mu\}=\operatorname{sign}\left\{\mathcal{R} e(d \lambda / d \mu)^{-1}\right\}$. Since $i \omega\left(\mu_{c}\right)$ is a purely imaginary root of (6.5), then, from (6.11), $\omega\left(\mu_{c}\right)^{2}=c^{2}-b^{2}$, and we obtain

$$
\begin{aligned}
& \operatorname{sign}\left\{\left.\frac{d \mathcal{R} e(\lambda)}{d \mu}\right|_{\mu=\mu_{c}}\right\} \\
& =\operatorname{sign}\left\{c\left[c^{3}\left(\mu_{c} \hat{\tau}_{c}^{\prime}+\tau_{c}^{*}\right)+c^{2} c^{\prime} \mu_{c} \tau^{*}-c\left(b^{2}\left(\mu_{c} \hat{\tau}_{c}^{\prime}+\tau_{c}^{*}\right)+b^{\prime}+b b^{\prime} \mu_{c} \tau_{c}^{*}\right)+c^{\prime} b\right]\right\} .
\end{aligned}
$$

As we already noticed, if (6.5) has pure imaginary roots, then necessarily $c>0$. We deduce (6.14), and the proof is complete.

In the next theorem we summarize and prove our results dealing with the asymptotic stability of the positive steady state $N^{*}$.

Theorem 6.6. Assume (6.2) holds true; $\beta$ and $\tau$ are $C^{2}$ functions. If no $\mu \in$ $[0, \bar{\mu})$ satisfies (6.8), then the positive steady state $N^{*}$ of (3.7) is locally asymptotically stable for $\mu \in[0, \bar{\mu})$.

Assume there exists $\mu^{*} \in(0, \bar{\mu})$ such that (6.8) is fulfilled for $\mu \in\left[0, \mu^{*}\right)$ (for instance, if (R1) and (R2) are fulfilled). Then, the following hold true:

(i) If $Z_{0}$, defined in (6.13), has no root on the interval $\left[0, \mu^{*}\right)$, then the positive steady state $N^{*}$ of (3.7) is locally asymptotically stable for $\mu \in\left[0, \mu^{*}\right)$.

(ii) If $Z_{0}$ has at least one positive root $\mu_{c} \in\left(0, \mu^{*}\right)$, then $N^{*}$ is locally asymptotically stable for $\mu \in\left[0, \mu_{c}\right)$ and unstable for $\mu \geq \mu_{c}$, $\mu$ in a neighborhood of $\mu_{c}$, and a Hopf bifurcation occurs at $N^{*}$ for $\mu=\mu_{c}$ if

$$
\begin{aligned}
& c^{3}\left(\mu_{c} \hat{\tau}_{c}^{\prime}+\tau_{c}^{*}\right)+c^{2} c^{\prime} \mu_{c} \tau_{c}^{*} \\
& -c\left(b^{2}\left(\mu_{c} \hat{\tau}_{c}^{\prime}+\tau_{c}^{*}\right)+b^{\prime}+b b^{\prime} \mu_{c} \tau_{c}^{*}\right)+c^{\prime} b \neq 0 .
\end{aligned}
$$

When (ii) holds true, several stability switches can potentially occur for every $\tau=\tau_{c}$, roots of $Z_{k}$ functions.

Proof. From Lemma 6.3 we know that $N^{*}$ is locally asymptotically stable when $\mu=0$. The first statement is straightforwardly satisfied.

Assume there exists $\mu^{*} \in(0, \bar{\mu})$ such that (6.8) is fulfilled for $\mu \in\left[0, \mu^{*}\right)$. If $Z_{0}$ has no positive root on the interval $\left(0, \mu^{*}\right)$, then the characteristic equation (6.5) has no pure imaginary root (see Remark 1 and Lemma 6.4). Consequently, the stability of $N^{*}$ cannot be lost when $\mu$ increases. We obtain the statement in (i).

Now, if $Z_{0}$ has at least one positive root, say, $\mu_{c} \in\left(0, \mu^{*}\right)$, then (6.5) has a pair of simple conjugate pure imaginary roots $\pm i \omega_{c}$ for $\mu=\mu_{c}$. From (6.16) together with Proposition 6.5, we have either $d \mathcal{R} e(\lambda) / d \mu\left(\mu=\mu_{c}\right)>0$ or $d \mathcal{R} e(\lambda) / d \mu\left(\mu=\mu_{c}\right)<0$. By contradiction, we assume there exists a branch of characteristic roots $\lambda(\mu)$ such that $\lambda\left(\mu_{c}\right)=i \omega_{c}$ and $d \mathcal{R} e(\lambda(\mu)) / d \mu<0$ for $\mu<\mu_{c}, \mu$ close to $\mu_{c}$. Then there exists a characteristic root $\lambda(\mu)$ such that $\mathcal{R} e(\lambda(\mu))>0$ and $\mu<\mu_{c}$. Since $N^{*}$ is locally asymptotically stable when $\mu=0$, applying Rouché's theorem [23], we obtain that all characteristic roots of (6.5) have negative real parts when $\mu \in\left[0, \mu_{c}\right)$, and we obtain a contradiction. Thus,

$$
\left.\frac{d \mathcal{R} e(\lambda)}{d \mu}\right|_{\mu=\mu_{c}}>0
$$

Now, let $N$ be a solution of (3.7). Then the function $x$, defined, for $t \geq 0$, by

$$
x(t):=N(\mu t),
$$


satisfies

$$
\dot{x}(t)=\zeta\left(\mu, x_{t}\right),
$$

where $\zeta: \mathbb{R}^{+} \times C \rightarrow \mathbb{R}$ is given by

$$
\zeta(\mu, \phi)=-\mu(\delta+\beta(\phi(0))) \phi(0)+2 \mu e^{-\gamma \mu \tilde{\tau}(\phi(0))} \beta(\phi(-\tilde{\tau}(\phi(0)))) \phi(-\tilde{\tau}(\phi(0))) .
$$

In order to prove the Hopf bifurcation for (3.7), from the change of variable in (6.18), it suffices to prove the Hopf bifurcation for (6.19). In [26], Eichmann stated some conditions on the function $\zeta$ allowing a Hopf bifurcation to occur at the positive steady state of (6.19). The function $\zeta$ defined above satisfies in particular the assumptions in [26], which are not recalled here for the sake of clarity.

The characteristic equation associated with the equilibrium $N^{*}$ of (6.19) is

$$
\begin{aligned}
\tilde{\Delta}(\lambda, \mu)= & \lambda+\mu(\delta+\bar{\beta})+\mu^{2} \tilde{\tau}^{\prime}\left(N^{*}(\mu)\right) \bar{\alpha} e^{-\gamma \mu \tilde{\tau}\left(N^{*}(\mu)\right)} \\
& -2 \mu \bar{\beta} e^{-\gamma \mu \tilde{\tau}\left(N^{*}(\mu)\right)} e^{-\lambda \tilde{\tau}\left(N^{*}(\mu)\right)} .
\end{aligned}
$$

Let $\lambda \in \mathbb{C}$. One can see that $\tilde{\Delta}(\mu \lambda, \mu)=\mu \Delta(\lambda, \mu)$ for all $\mu \in[0, \bar{\mu})$. Then, for $\mu \in(0, \bar{\mu}), \lambda$ is an eigenvalue associated with (6.20) if and only if $\lambda / \mu$ is an eigenvalue associated with (6.5). Thus, it is straightforward that (6.20) has a unique pair of simple conjugate purely imaginary eigenvalues for $\mu=\mu_{c}$, which satisfy (6.17), since these properties hold for the characteristic equation (6.5). Then, from [26], a Hopf bifurcation occurs at $N^{*}$ when $\mu=\mu_{c}$. This concludes the proof.

Remark 2. The asymptotic stability of the positive steady state of (3.7) has been analyzed with respect to the parameter $\mu$. One may notice that it can, however, be investigated with respect to every parameter in the model. The role of each parameter on the stability of the positive steady state is numerically determined in the next section, which is devoted to numerical simulations of solutions of (3.7).

7. Numerical simulations. Inspired by Mackey [43], Pujo-Menjouet and Mackey [53], and Adimy, Crauste, and Ruan [5], we take $\beta$ as a Hill function, given by (2.2). The following values for the parameters are chosen according to $[43,52,53]$ :

$$
\delta=0.05 \text { day }^{-1}, \quad \gamma=0.2 \text { day }^{-1}, \quad \beta_{0}=1.77 \text { day }^{-1}, \quad \text { and } \quad r=3 .
$$

The value of $\theta$ is set to $\theta=1$ cell.g $^{-1}$, which is not relevant for a quantitative analysis, but does not modify the qualitative behavior of solutions of (3.7).

The delay function $\tilde{\tau}$ is chosen as

$$
\tilde{\tau}(N)=\tilde{\tau}_{0}+\left(\tilde{\tau}_{\max }-\tilde{\tau}_{0}\right) \frac{N}{N+\theta_{\tau}},
$$

with $\tilde{\tau}_{\max }>\tilde{\tau}_{0}>0$ and $\theta_{\tau}>0$ (otherwise the delay is constant). Hence $\tilde{\tau}(N)$, and consequently $\tau(N)$, is an increasing bounded positive function. One may note that $\tau_{0}=\mu \tilde{\tau}_{0}$ and $\tau_{\max }=\mu \tilde{\tau}_{\max }$.

We use MATLAB and the solver DDESD [56] for state-dependent delay differential equations to numerically compute the solutions of (3.7).

In a first example, we fix

$$
\tilde{\tau}_{0}=0.1 \text { day, } \quad \tilde{\tau}_{\max }=1 \text { day, } \quad \text { and } \quad \theta_{\tau}=1 \text { cell.g }{ }^{-1} .
$$

With values in (7.1) and (7.2), a unique positive steady state $N^{*}(\mu)$ of (3.7) exists (condition (4.1) holds true) for $\mu<\bar{\mu}$, where computations give $\bar{\mu} \approx 33$ (see Figure 


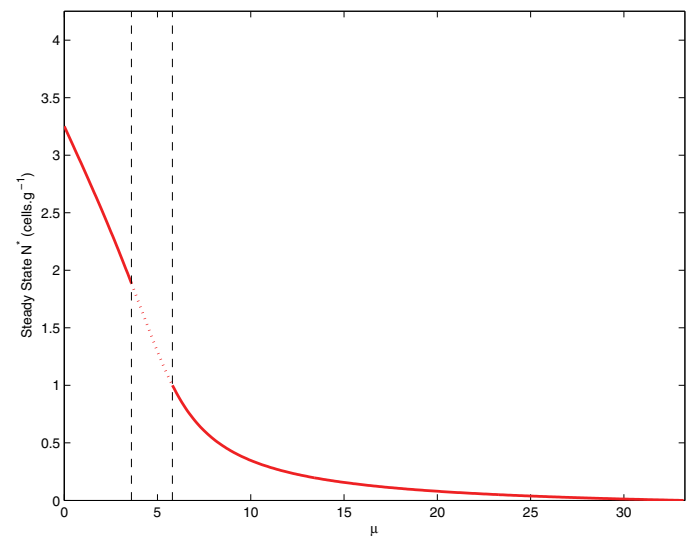

FIG. 7.1. Positive steady state $N^{*}(\mu)$ of (3.7) for $\mu \in[0, \bar{\mu})$. The solid line corresponds to stable values of the steady state, whereas the dotted lines account for unstable values of $N^{*}(\mu)$. Parameters are given by (7.1) and (7.2).
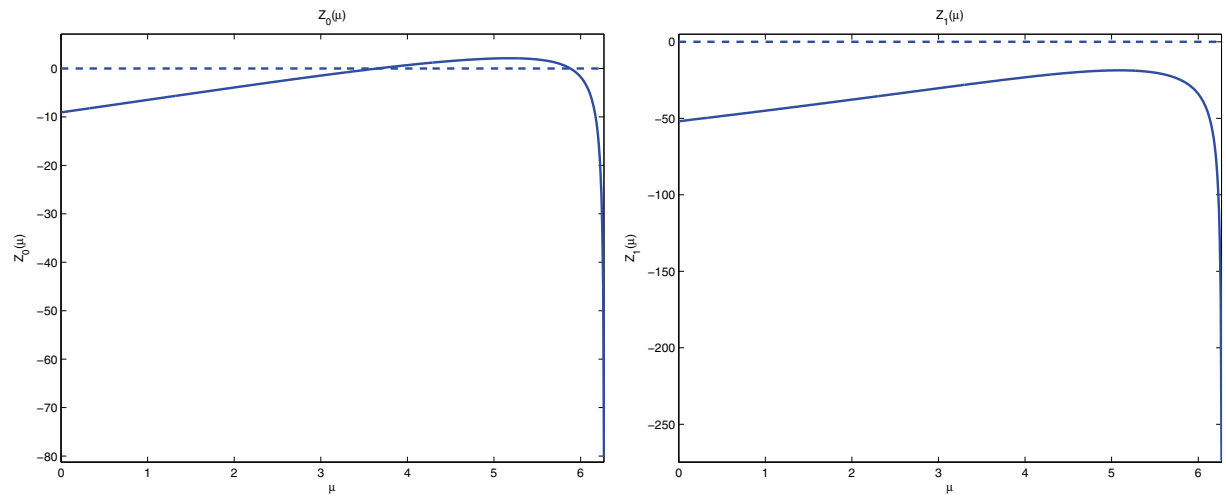

Fig. 7.2. Functions $Z_{k}$. Left: Function $Z_{0}$, which exhibits two positive roots, $\mu_{1}=3.66$ and $\mu_{2}=5.88$. Right: Function $Z_{1}$, which is negative. Parameters are given by (7.1) and (7.2).

7.1). We also obtain $\mu^{*}=6.27$ and the functions $Z_{k}$ defined in Lemma 6.4. The function $Z_{0}$ has two roots in $\left(0, \mu^{*}\right), \mu_{1}=3.66$, and $\mu_{2}=5.88$, and the function $Z_{1}$ has no root. This is displayed in Figure 7.2.

According to the results stated in Theorem 6.6, the positive steady state $N^{*}$ is asymptotically stable for $\mu<\mu_{1}$ and $\mu>\mu_{2}$ and unstable when $\mu \in\left[\mu_{1}, \mu_{2}\right]$. In this latter case, $N^{*}$ undergoes a Hopf bifurcation when $\mu=\mu_{1}$, and the solutions of (3.7) periodically oscillate with a period of about 17 days. This is shown in Figure 7.3. Variations of the delay function $\tilde{\tau}(N(t))$ are also displayed. The same periodic behavior is observed.

The stability and instability areas, delimited by the roots of the $Z_{k}$ functions, can be computed as functions of a given parameter. For instance, critical values of $\mu$ as functions of $\theta_{\tau}$ are displayed in Figure 7.4. The stability zone is bounded above by the value $\bar{\mu}$. For $\mu \geq \bar{\mu},(3.7)$ has only one steady state, $N \equiv 0$. The stability area decreases as $\theta_{\tau}$ increases. When $\theta_{\tau}$ becomes very large, the instability zone is a strip with rather constant width, and, as $\mu$ increases, one always observes a stability switch (from unstable to stable) before the positive steady state no longer exists, that is, for 

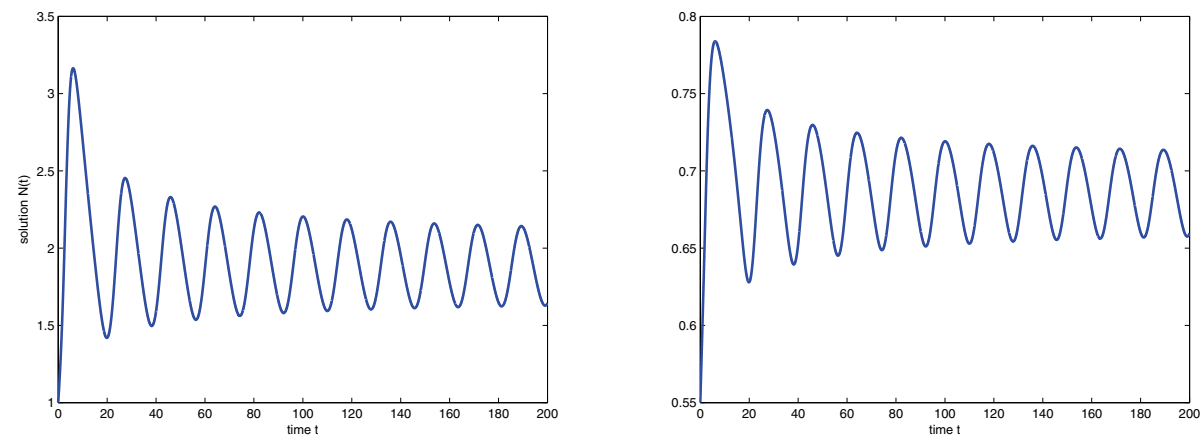

Fig. 7.3. Left: Periodic solution at the Hopf bifurcation. With parameters given by (7.1) and (7.2), the solution of (3.7) undergoes a Hopf bifurcation when $\mu=3.66$, so periodic solutions are observed. Right: The corresponding delay function $\tilde{\tau}(N(t))$.

$\mu>\bar{\mu}$ (not shown here). One can also notice that when $\theta_{\tau}=0$ a stability switch appears as $\mu$ increases. In this case, the delay is constant, with $\tau(N)=\mu \tilde{\tau}_{\max }=\tau_{\max }$. Hence the instability area appears to be larger when the delay depends on the cell population than in the constant case with a delay $\tau=\tau_{\max }$.

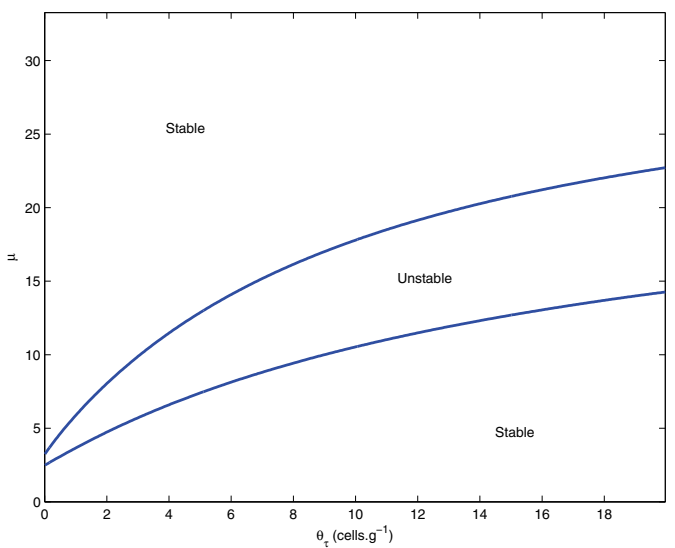

FIG. 7.4. With parameters given by (7.1) and (7.2), except for $\theta_{\tau}$ which varies, the stable and unstable zones in the $\left(\theta_{\tau}, \mu\right)$-plane are obtained.

Critical values of $\mu$, as functions of $\tilde{\tau}_{0}$ and $\tilde{\tau}_{\max }$, are presented in Figure 7.5. Contrary to $\theta_{\tau}$, values of $\tilde{\tau}_{0}$ and $\tilde{\tau}_{\max }$ for which the positive steady state exists are limited: $\tilde{\tau}_{0}$ must satisfy (4.1) and be less than $\tilde{\tau}_{\max }$. One can observe that instability areas are rather small compared to the stability areas, both in the $\left(\tilde{\tau}_{0}, \mu\right)$-plane and in the $\left(\tilde{\tau}_{\max }, \mu\right)$-plane. The dependence of critical values of $\mu$ on $\tilde{\tau}_{\max }$ seems strong: one observes in Figure 7.5 that values of $\mu$ for which stability switch occurs drastically decrease when $\tilde{\tau}_{\max }$ increases. For large values of $\tilde{\tau}_{\max }$, the positive steady state $N^{*}(\mu)$ is almost always stable. However, for every value of $\tilde{\tau}_{0}, \tilde{\tau}_{\max }$, and $\theta_{\tau}$ there exist values of $\mu$ for which the steady state is unstable. This is not the case with all parameters, as described hereafter.

In Figure 7.6, critical values of $\mu$ are displayed as functions of $\gamma$ and $r$, defined 

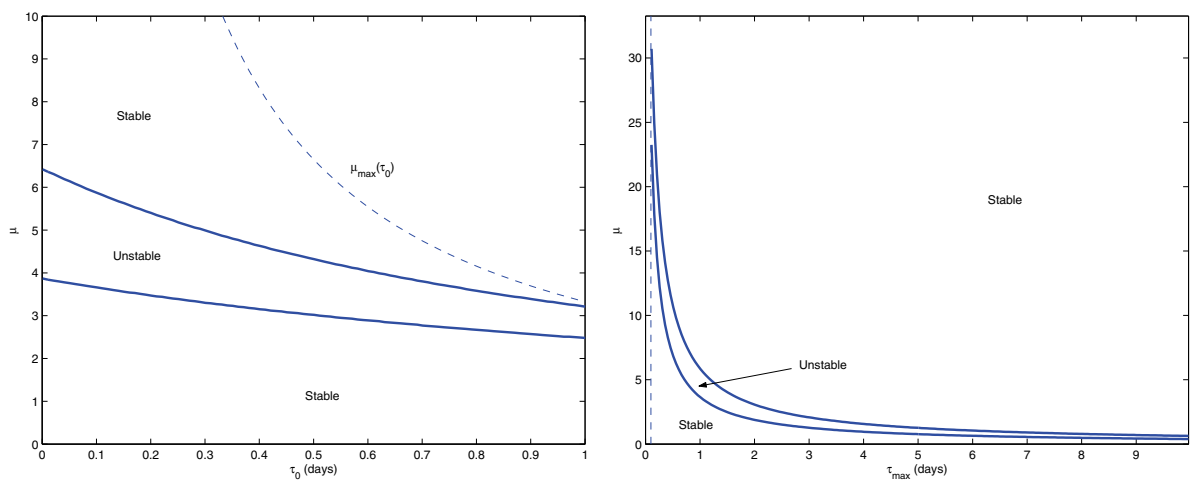

FIG. 7.5. Left: Critical values of $\mu\left(\tilde{\tau}_{0}\right)$, with $\mu<\bar{\mu}\left(\tilde{\tau}_{0}\right)$, for $\tilde{\tau}_{0}$ satisfying (4.1) and $\tilde{\tau}_{0}<\tilde{\tau}_{\max }$. Parameters are given by (7.1) and (7.2), except for $\tilde{\tau}_{0}$ which varies. Right: Critical values of $\mu\left(\tilde{\tau}_{\max }\right)$ for $\tilde{\tau}_{\max }$ satisfying $\tilde{\tau}_{0}<\tilde{\tau}_{\max }$. Parameters are given by (7.1) and (7.2), except for $\tilde{\tau}_{\max }$ which varies.
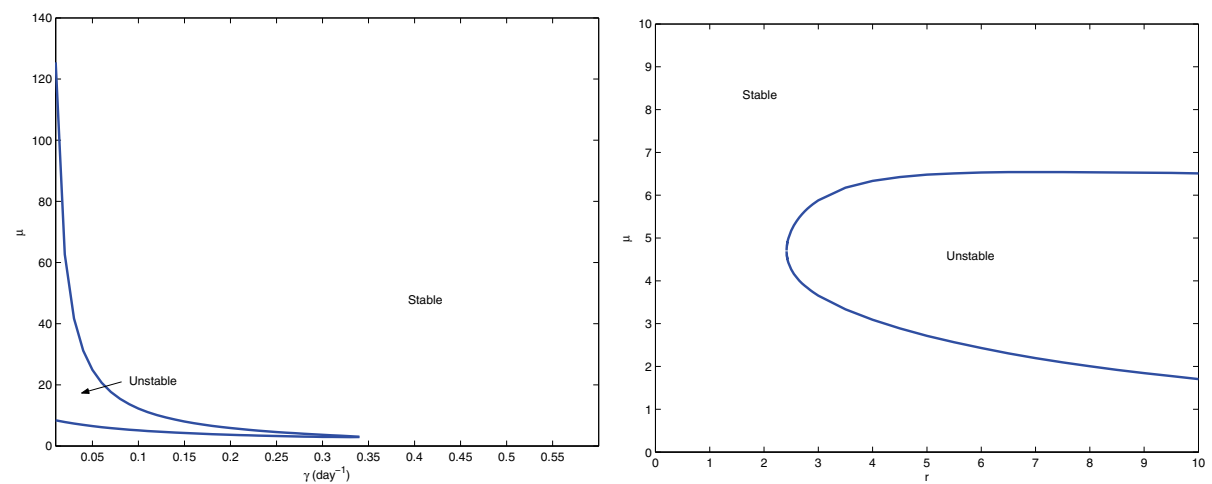

FIG. 7.6. Left: Critical values of $\mu(\gamma)$, with parameters given by (7.1) and (7.2), except for $\gamma$ which varies. Right: Critical values of $\mu(r)$, with parameters given by (7.1) and (7.2), except for $r$ which varies.

in (2.2), respectively. Critical values of $\delta$ (resp., $\beta_{0}$ ) are not shown here, yet they are qualitatively similar to the ones obtained for $\gamma$ (resp., $r$ ). Differences are mentioned in the following discussion.

The instability zone is limited in the $(\gamma, \mu)$ - or $(\delta, \mu)$-plane and appears rather small compared to the stability zone. For small values of $\gamma$, close to zero, a wide range of values of $\mu$ induces instability of the solution. The influence of $\gamma$ on the stability of $N^{*}(\mu)$ seems strong, especially small values of $\gamma$, that is, low apoptosis rates of proliferating cells. High apoptosis rates correspond to a stable steady state.

For small values of $\beta_{0}$ and $r$ the positive steady state is always stable. As $r$ increases (that is, the sensitivity of the introduction rate becomes more important), the width of the instability area increases. Hence, a large sensitivity $r$ is likely to induce instability. This is not the case with $\beta_{0}$ (not shown here), whose variations do not increase the instability range.

This numerical study of the stability of the positive steady state $N^{*}(\mu)$ indicates that three categories of parameters play crucial roles in the stability of the system. First, all parameters related to the delay function $\tilde{\tau}(N)$, that is, $\tilde{\tau}_{0}, \tilde{\tau}_{\max }$, and $\theta_{\tau}$, 
induce, as they increase, stability switches of the positive steady state. For every combination of $\left(\tilde{\tau}_{0}, \tilde{\tau}_{\max }, \theta_{\tau}\right)$ it is possible to find values of $\mu$ for which the steady state is unstable. This is not surprising, since the delay has been shown, in Theorem 6.6, to lead to instability through the variations of $\mu$. The parameters defining the delay function are consequently expected to modify the stability of the system.

Second, the apoptosis rate $\gamma$ was shown to induce stability switches, particularly for small values. This observation had already been noticed in previous works, with constant delay. See, for instance, Mackey [43], Pujo-Menjouet and Mackey [53], PujoMenjouet, Bernard, and Mackey [52], and Adimy and Crauste [3].

Finally, the sensitivity $r$ of the introduction rate $\beta(N)$ was shown to play a significant role in the instability of the positive steady state for large values. This sensitivity is related to the ability of cells to react to stimuli that induce their activation [43]. This indicates that an increase in the ability for a cell to be activated is likely to trigger periodic behaviors.

Instability of the steady state corresponds to oscillating solutions (since solutions are bounded). Hence, it appears relevant to investigate the influence of the parameters involved in the problem on periods and amplitudes of the oscillations observed when the steady state is unstable.

We first investigate the influence of the parameters related to the delay function, that is, $\mu, \tilde{\tau}_{0}$, and $\tilde{\tau}_{\max }$. We fix all the parameters except the one we focus on, which ranges in what we shall call its instability range. This is the domain (in what follows, an interval), associated with the fixed parameter values, in which the free parameter can range to ensure instability of the steady state. For instance, when all the parameters are given by (7.1) and (7.2), the instability range of $\mu$ is $\left[\mu_{1}, \mu_{2}\right]$, with $\mu_{1}=3.66$ and $\mu_{2}=5.88$. For parameters different from $\mu$, the instability range is easily obtained from Figures 7.5 and 7.6 by fixing $\mu$ to a given value (hereafter, $\mu=4.2$ is used) and determining on the graph the limiting values of the parameter. From Figure 7.6, for instance, the instability range of $\gamma$ is deduced; it is equal to $[0.15,0.27]$.

In Figure 7.7, periods and amplitudes of the oscillations are displayed when $\tilde{\tau}_{0}$ varies in its instability range. Variations of $\mu$ and $\tilde{\tau}_{\max }$ are not shown here, yet they are similar.

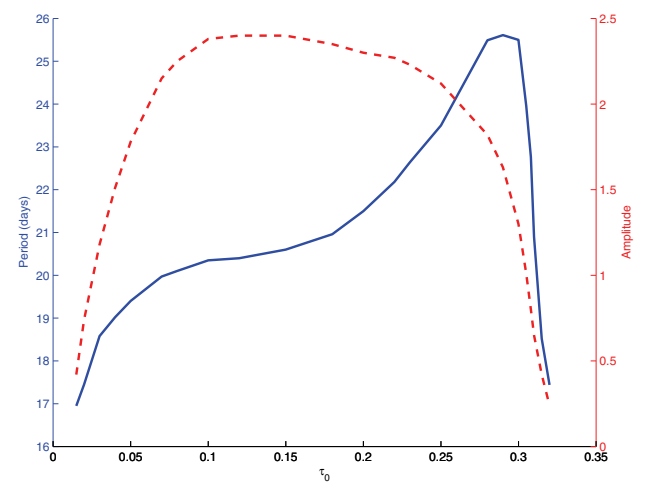

FIG. 7.7. Periods (solid line) and amplitudes (dashed line) of oscillating solutions, as functions of $\tau_{0}$. All other parameters are fixed, given by (7.1) and (7.2), and $\mu=4.2$. Scale of the periods is on the left axis; scale of the amplitudes is on the right axis.

The three parameters have almost the same influence on amplitudes of the os- 
cillations, with the same variations. One can consider that amplitudes do not really vary: they quickly reach some plateau, between 2 and 2.5 , and finally decrease rapidly before the stability switch. Their influence on the periods of oscillating solutions is more important although limited. Three windows appear when looking at the evolution of periods: a first rapid increase of the periods, then a plateau of about $20-21$ days, and then another increase that does not reach high values. Then, even though the influence of the three parameters related to the delay function on the stability of the steady state has been noticed above, their contribution to period and amplitude variations is very limited (especially for amplitude variations).

In Figure 7.8, periods and amplitudes of oscillating solutions are shown as functions of $\gamma$ and $r$. Graphs of periods and amplitudes of oscillating solutions as functions of $\delta$ (resp., $\beta_{0}$ ) are not shown here but are similar to the graphs as functions of $\gamma$ (resp., $r$ ).
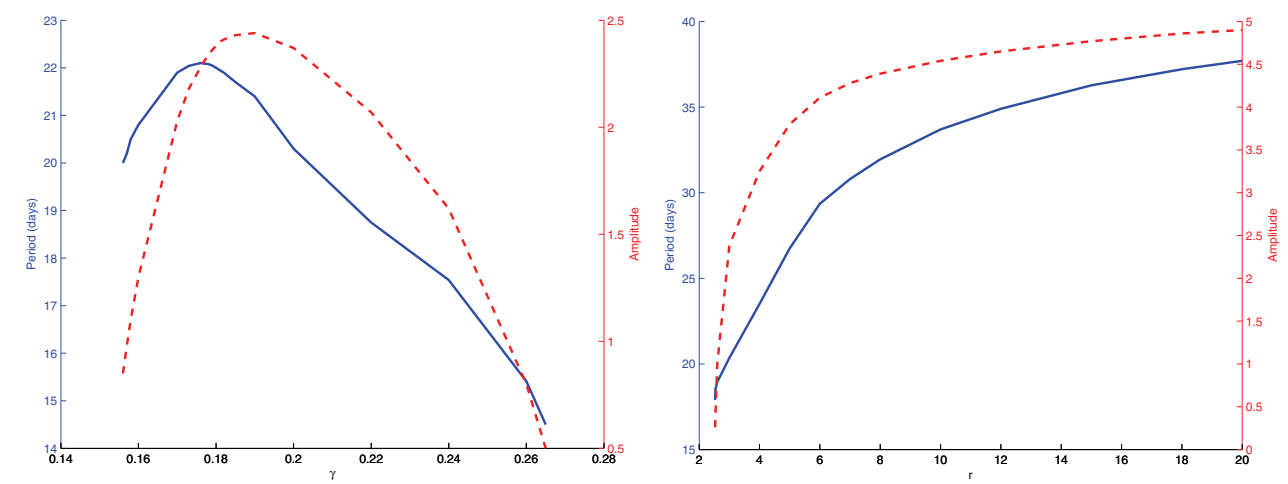

FIG. 7.8. Periods (solid lines) and amplitudes (dashed lines) of oscillating solutions, as functions of $\gamma$ (left) and $r$ (right). For each figure, all other parameters are fixed, given by (7.1) and (7.2), and $\mu=4.2$. Scale of the periods is on the left axis; scale of the amplitudes is on the right axis.

Two different types of parameters are identified in Figure 7.8: those that tend to decrease periods and amplitudes of the oscillations, $\gamma$ and $\delta$, and those that increase them, $\beta_{0}$ and $r$. One has to note that parameters $\beta_{0}$ and $r$ are not bounded above, their instability ranges are not limited, and they can then increase far from biologically relevant values. However, their positive action on periods and amplitudes are established, and one can easily check that maximal values obtained with other parameters $\left(\mu, \tilde{\tau}_{\max }, \tilde{\tau}_{0}\right)$ are quickly overtaken. For instance, periods larger than 25 days are obtained as soon as $r \geq 5$, and amplitudes are larger than 2.5 for $r \geq 3$.

The decreasing action of mortality rates is very significant. Instability usually occurs for small values of mortality rates, and the numerical results in Figure 7.8 clearly show that qualitative properties of oscillating solutions are also strongly affected by variations of mortality rates.

Finally, two categories of parameters emerge: those favoring an increase of periods and amplitudes of oscillating solutions, $\beta_{0}$ and $r$, and those decreasing both periods and amplitudes, $\gamma$ and $\delta$. The first category of parameters is associated with cell proliferation, whereas the second one is formed with mortality rates. The parameters associated with the delay, that is, $\mu, \tilde{\tau}_{\max }$, and $\tilde{\tau}_{0}$, have limited influence. They have almost no influence on the amplitudes of oscillations, and they act similarly on periods of oscillations. 
The influence of the parameters on the stability of the unique positive steady state have already been analyzed by Pujo-Menjouet, Bernard, and Mackey [52] for the discrete model (that is, with $\tau$ constant) with a constant introduction rate $\beta$. They found that $\beta$ and $\tau$ mainly influenced amplitudes of oscillations, and $\delta$ and $\gamma$ influenced periods of oscillations.

Our results, for a different model in which the nature of the delay modifies the roles of the parameters, gives different results. The main difference is that parameters related to the delay have not been found to influence either periods or amplitudes of oscillating solutions. Yet, they were shown to play an important role in the stability of the steady state, which was expected from the nature of the delay.

Acknowledgment. The authors are grateful to the anonymous referees for their valuable and helpful comments that improved this manuscript.

\section{REFERENCES}

[1] M. Adimy And F. Crauste, Global stability of a partial differential equation with distributed delay due to cellular replication, Nonlinear Anal., 54 (2003), pp. 1469-1491.

[2] M. Adimy And F. CRAuste, Existence, positivity and stability for a nonlinear model of cellular proliferation, Nonlinear Anal. Real World Appl., 6 (2005), pp. 337-366.

[3] M. Adimy AND F. CRauste, Mathematical model of hematopoiesis dynamics with growth factor-dependent apoptosis and proliferation regulations, Math. Comput. Modelling, 49 (2009), pp. 2128-2137.

[4] M. Adimy, F. Crauste, and L. Pujo-Menjouet, On the stability of a nonlinear maturity structured model of cellular proliferation, Discrete Contin. Dyn. Syst., 12 (2005), pp. 501522.

[5] M. Adimy, F. Crauste, and S. Ruan, A mathematical study of the hematopoiesis process with applications to chronic myelogenous leukemia, SIAM J. Appl. Math., 65 (2005), pp. $1328-1352$.

[6] M. Adimy, F. Crauste, and S. Ruan, Stability and Hopf bifurcation in a mathematical model of pluripotent stem cell dynamics, Nonlinear Anal. Real World Appl., 6 (2005), pp. 651670.

[7] M. Adimy And L. Pujo-Menjouet, A singular transport model describing cellular division, C. R. Acad. Sci. Paris Ser. I Math., 332 (2001), pp. 1071-1076.

[8] M. Adimy and L. Pujo-Menjouet, A mathematical model describing cellular division with a proliferating phase duration depending on the maturity of cells, Electron. J. Differential Equations, 107 (2003), pp. 1-14.

[9] W. G. Aiello, H. I. Freedman, and J. Wu, Analysis of a model representing stage-structured population growth with state-dependent time delay, SIAM J. Appl. Math., 52 (1992), pp. $855-869$.

[10] O. Arino, K. P. Hadeler, And M. L. HBid, Existence of periodic solutions for delay differential equations with state dependent delay, J. Differential Equations, 144 (1998), pp. 263-301.

[11] O. Arino And E. Sanchez, A saddle point theorem for functional state-dependent delay equations, Discrete Contin. Dyn. Syst., 12 (2005), pp. 687-722.

[12] T. Alarcon And M. J. Tindall, Modelling cell growth and its modulation of the G1/S transition, Bull. Math. Biol., 69 (2007), pp. 197-214.

[13] J. BÉLAIR, Population models with state-dependent delays, in Mathematical Population Dynamics (New Brunswick, NJ, 1989), Lecture Notes in Pure and Appl. Math. 131, Dekker, New York, 1991, pp. 165-176.

[14] J. Bélair, M. C. Mackey, and J. M. Mahaffy, Age-structured and two-delay models for erythropoiesis, Math. Biosci., 128 (1995), pp. 317-346.

[15] E. Beretta And Y. Kunng, Geometric stability switch criteria in delay differential systems with delay dependent parameters, SIAM J. Math. Anal., 33 (2002), pp. 1144-1165.

[16] S. Bernard, J. Belair, ANd M. C. Mackey, Sufficient conditions for stability of linear differential equations with distributed delay, Discrete Contin. Dyn. Syst., 1 (2001), pp. 233-256.

[17] F. J. Burns And I. F. TANnock, On the existence of a $G_{0}$ phase in the cell cycle, Cell. Tissue Kinet., 19 (1970), pp. 321-334. 
[18] S. H. Cheshier, S. J. Morrison, X. Liao, and I. L. Weissman, In vivo proliferation and cell cycle kinetics of long-term self-renewing hematopoietic stem cells, Proc. Natl. Acad. Sci. USA, 96 (1999), pp. 3120-3125.

[19] K. Cooke And W. Huang, On the problem of linearization for state-dependent delay differential equations, Proc. Amer. Math. Soc., 124 (1996), pp. 1417-1426.

[20] F. CRAuste, Global asymptotic stability and Hopf bifurcation for a blood cell production model, Math. Biosci. Engrg., 3 (2006), pp. 325-346.

[21] F. Crauste, Delay model of hematopoietic stem cell dynamics: Asymptotic stability and stability switch, Mathematical Modelling of Natural Phenomena, 4 (2009), pp. 28-47.

[22] F. Crauste, L. Pujo-Menjouet, S. Genieys, C. Molina, and O. Gandrillon, Adding selfrenewal in committed erythroid progenitors improves the biological relevance of a mathematical model of erythropoiesis, J. Theoret. Biol., 250 (2008), pp. 322-338.

[23] J. Dieudonné, Foundations of Modern Analysis, Academic Press, New York, 1960.

[24] R. D. Driver, Existence theory for a delay-differential system, Contrib. Differential Equations, 1 (1963), pp. 317-336.

[25] J. Dyson, R. Villella-Bressan, and G. F. WebB, A singular transport equation modelling a proliferating maturity structured cell population, Canad. Appl. Math. Quart., 4 (1996), pp. 65-95.

[26] M. Eichmann, A Local Hopf Bifurcation Theorem for Differential Equations with StateDependent Delays, Ph.D. thesis, 2006.

[27] P. Fortin And M. C. Mackey, Periodic chronic myelogenous leukemia: Spectral analysis of blood cell counts and etiological implications, Brit. J. Haematol., 104 (1999), pp. 336-345.

[28] H. Fuss, W. Dubitzky, S. Downes, and M. J. Kurth, Mathematical models of cell cycle regulation, Brief Bioinform., 6 (2005), pp. 163-177.

[29] J. K. Hale, Theory of Functional Differential Equations, Springer-Verlag, Berlin, 1977.

[30] F. HARTUNG, Linearized stability in periodic functional differential equations with statedependent delays, J. Comput. Appl. Math., 174 (2005), pp. 201-211.

[31] F. HaRtung AND J. TURI, Linearized stability in functional differential equations with statedependent delays, Discrete Contin. Dynam. Systems, added volume (2001), pp. 416-425.

[32] F. Hartung, T. Krisztin, H. O. Walther, and J. Wu, Functional differential equations with state-dependent delay: Theory and applications, in Handbook of Differential Equations: Ordinary Differential Equations Volume 3, A. Canada, P. Drabek, and A. Fonda eds., Elsevier Science B. V., North-Holland, Amsterdam, 2006, pp. 435-545.

[33] C. Haurie, D. C. Dale, and M. C. Mackey, Cyclical neutropenia and other periodic hematological diseases: A review of mechanisms and mathematical models, Blood, 92 (1998), pp. 2629-2640.

[34] M. L. Hbid, E. SAnchez, And R. Bravo DE LA PARRA, State-dependent delays associated to threshold phenomena in structured population dynamics, Math. Models Methods Appl. Sci., 17 (2007), pp. 877-900.

[35] E. P. Hofer, B. Tibken, And F. Lehn, Differential equations with state-dependent delays, in Online Optimization of Large Scale Systems, Springer, Berlin, 2001, pp. 413-432.

[36] E. P. Hofer, B. Tibken, And F. Lehn, Biomathematical models with state-dependent delays for granulocytopoiesis, in Online Optimization of Large Scale Systems, Springer, Berlin, 2001, pp. 433-453.

[37] Y. I. Kazmerchuk AND J. Wu, Stochastic state-dependent delay differential equations with applications in finance, Funct. Differ. Equ., 11 (2004), pp. 77-86.

[38] Y. Kunang, Delay Differential Equations with Application in Population Dynamics, Mathematics in Science and Engineering 191, Academic Press, New York, 1993.

[39] Y. KuAng And H. L. SMith, Slowly oscillating periodic solutions of autonomous statedependent delay differential equations, Nonlinear Anal., 19 (1992), pp. 855-872.

[40] L. G. Lajtha, On DNA labeling in the study of the dynamics of bone marrow cell populations, in The Kinetics of Cellular Proliferation, F. Stohlman, Jr., ed., Grune and Stratton, New York, 1959, pp. 173-182.

[41] M. Louihi, M. L. HBid, And O. ARino, Semigroup properties and the Crandall-Liggett approximation for a class of differential equations with state-dependent delays, J. Differential Equations, 181 (2002), pp. 1-30.

[42] N. MacDonald, Time Lags in Biological Models, Lecture Notes in Biomathematics 27, Springer-Verlag, New York, 1978.

[43] M. C. MACKEY, Unified hypothesis of the origin of aplastic anemia and periodic hematopoiesis, Blood, 51 (1978), pp. 941-956. 
[44] M. C. Mackey, Dynamic hematological disorders of stem cell origin, in Biophysical and Biochemical Information Transfer in Recognition, J. G. Vassileva-Popova and E. V. Jensen, eds., Plenum Press, New York, 1979, pp. 941-965.

[45] M. C. MACKey AND A. ReY, Multistability and boundary layer development in a transport equation with retarded arguments, Canad. Appl. Math. Quart., 1 (1993), pp. 1-21.

[46] M. C. MACKEY AND R. RUDNICKI, Global stability in a delayed partial differential equation describing cellular replication, J. Math. Biol., 33 (1994), pp. 89-109.

[47] M. C. Mackey AND R. Rudnicki, A new criterion for the global stability of simultaneous cell replication and maturation processes, J. Math. Biol., 38 (1999), pp. 195-219.

[48] P. Magal and O. Arino, Existence of periodic solutions for state dependent delay differential equations, J. Differential Equations, 165 (2000), pp. 61-95.

[49] J. M. MAhaffy, J. BÉLAiR, AND M. C. MaCKey, Hematopoietic model with moving boundary condition and state dependent delay: Applications in erythropoiesis, J. Theoret. Biol., 190 (1998), pp. 135-146.

[50] J. Mallet-Paret, R. D. Nussbaum, and P. Paraskevopoulos, Periodic solutions for functional differential equations with multiple state-dependent time lags, Topol. Methods Nonlinear Anal., 3 (1994), pp. 101-162.

[51] R. Ouifki And M. L. HBID, Periodic solutions for a class of functional differential equations with state-dependent delay close to zero, Math. Models Meth. Appl. Sci., 13 (2003), pp. 807-841.

[52] L. Pujo-Menjouet, S. Bernard, and M. C. Mackey, Long period oscillations in a $G_{0}$ model of hematopoietic stem cells, SIAM J. Appl. Dyn. Syst., 4 (2005), pp. 312-332.

[53] L. Pujo-Menjouet and M. C. Mackey, Contribution to the study of periodic chronic myelogenous leukemia, C. R. Biologies, 327 (2004), pp. 235-244.

[54] A. V. Rezounenko And J. Wu, A non-local PDE model for population dynamics with stateselective delay: Local theory and global attractors, J. Comput. Appl. Math., 190 (2006), pp. 99-113.

[55] I. RuPES, Checking cell size in yeast, Trends Genet., 18 (2002), pp. 479-485.

[56] L. F. Shampine, Solving ODEs and DDEs with residual control, Appl. Numer. Math., 52 (2005), pp. 113-127.

[57] H. L. Smith And Y. Kuang, Periodic solutions of differential delay equations with thresholdtype delays, in Oscillation and Dynamics in Delay Equations, Contemporary Mathematics 129, J. R. Graef and J. K. Hale, eds., AMS, Providence, RI, 1992, pp. 153-176.

[58] J. J. TySOn And B. Novak, Regulation of the eukaryotic cell cycle: Molecular antagonism, hysteresis, and irreversible transitions, J. Theoret. Biol., 210 (2001), pp. 249-263.

[59] H. O. Walther, Stable periodic motion of a system with state-dependent delay, Differential Integral Equations, 15 (2002), pp. 923-944.

[60] H. O. WALThER, The solution manifold and C1-smoothness of solution operators for differential equations with state dependent delay, J. Differential Equations, 195 (2003), pp. 46-65.

[61] H. O. Walther, On a model for soft landing with state-dependent delay, J. Dynam. Differential Equations, 19 (2007), pp. 593-622.

[62] G. F. WebB, Theory of Nonlinear Age-Dependent Population Dynamics, Monographs and Textbooks in Pure and Applied Mathematics 89, Marcel Dekker, New York, 1985. 